\title{
The effect of early weaning on iron status among exclusively breastfed infants
}

\begin{abstract}
Background and objective: Exclusive breastfeeding is recommended for all newborns to gain optimal physical and neurodevelopmental growth. Despite all its advantages, prolonged breastfeeding alone without weaning places the child at risk of iron deficiency and iron deficiency anemia, which is the commonest micronutrient deficiency worldwide among infants. We investigated the effect of early weaning on the iron status of infants.

Methods: We selected 100 infants; all of them were six months old. Fifty of them were exclusively breastfed for four months and started weaning between four to six months while continued to breastfeed till the age of six months (early-weaning group). Another 50 infants were exclusively breastfed for six months without starting weaning (no-weaning group). Then we investigated both groups at the age of six months for both iron and hematological studies.

Results: In the present study, both study groups shared some similarities. In both groups, females accounted for more than half of the cases, the majority of the infants were residing in the urban areas, and most of the infants were delivered by cesarean section. The final result of the iron study and hematological parameters in the no-weaning group was significantly lower as compared to the early-weaning group. Both serum ferritin and serum iron were $7.42 \mathrm{ng} / \mathrm{ml}$ and $25.66 \mu \mathrm{g} / \mathrm{dl}$, respectively, in the no-weaning group and were $23.94 \mathrm{ng} / \mathrm{ml}$ and $46.98 \mu \mathrm{g} / \mathrm{dl}$ respectively in the early-weaning group. Hemoglobin level was $10.84 \mathrm{gm} / \mathrm{dl}$ in the no-weaning group compared to $11.54 \mathrm{gm} / \mathrm{dl}$ in the early-weaning group. Conclusion: We concluded that early weaning had a great role in the prevention of both iron deficiency and iron deficiency anemia in exclusively breastfed infants.
\end{abstract}

Keywords: Exclusive breastfeeding; Early weaning; Iron status.

\section{Introduction}

It is necessary for all infants to take proper and sufficient food to get perfect physical and mental health in their entire life. The type of food should be exclusive breast milk for the first six months of postnatal life which is rich in all the essential micronutrients that they need. From six months onward, complementary foods should be added to their foods while continuing breastfeeding for at least to the age of twelve months. ${ }^{1}$ Despite the policy of preventable measures in the last decade still, nutritional deficiencies remain the leading cause of morbidity and mortality in children worldwide. These children may be deficient in many micronutrients or a single essential nutrient like iron, vitamin A, folic acid, and zinc. Therefore, both quantity and quality of all nutrients are needed to avoid any nutritional deficiency. ${ }^{2}$ Iron deficiency is the commonest micronutrient deficiency worldwide, especially among those who are susceptible to it, including infants, toddlers, adolescents, and pregnant women, due to their relatively rapid growth and its increased demand. The prevalence is $40 \%$ in children under five years and $38 \%$ in pregnant women. ${ }^{3}$ Iron deficiency should be treated properly; otherwise, it will lead to iron deficiency anemia. Then, both iron deficiency and iron

${ }^{1}$ Raparin Pediatric Hospital, Erbil, I raq

2 Department of Pediatrics, College of Medicine, Hawler Medical University, Erbil, I raq.

* Correspondence: kayfeekayfi@gmail.com 
deficiency anemia may adversely affect cognitive development in infants and children. $^{4-6}$ Iron deficiency has many adverse effects on the physiological functions of different tissues like the brain, blood, and muscles, depending on its severity. In the early stage, only iron stores are depleted without anemia. However, physical performance may still be affected. Then iron deficiency, if lasted long enough, erythropoiesis is affected without hemoglobin depletion, and finally, it leads to anemia. ${ }^{7}$ Nutritional deficiency remains the most common cause of iron deficiency anemia, especially in developing countries. $^{3}$ The young term infants do notdevelop iron deficiency anemia in the first four months because they are born with enough iron store during their intrauterine life that is sufficient for four to six months of postnatal life. Young infants grow rapidly, which leads to increased nutritional requirements, including iron, to fulfill high metabolic rate and blood volume expansion. Utilizing iron in high amounts leads to a decrease in its store significantly in four months of age. ${ }^{8}$ Compared to cow's milk, breast milk has good bioavailability of iron but insufficient in the amount of iron to meet the infant's need. ${ }^{6,9}$ Due to all its advantages on decreasing morbidity and mortality, exclusive breastfeeding for six months is recommended by many worldwide organizations, including the World Health Organization (WHO), to all newborns. ${ }^{1}$ However, it is still not followed by all nations and organizations; for example, in the United States, complementary foods are recommended to be started between four to six months by the US Department of Agriculture and the Centers for Disease Control (CDC). ${ }^{5}$ Besides all advantages of exclusive breastfeeding, some studies demonstrated that prolonged exclusive breastfeeding is associated with iron deficiency. Some others explained the advantages of early weaning between four to six months on iron status. ${ }^{10-12}$ Some others showed no difference between iron status and the duration of exclusive breastfeeding. ${ }^{13}$ Iron deficiency in six months old is defined as serum ferritin concentration of less than $12 \mathrm{mg} / \mathrm{dl}$. Iron deficiency anemia is defined as a hemoglobin level less than $11 \mathrm{gm} / \mathrm{dl}$ associated with iron deficiency. ${ }^{5}$ This study aimed to assess if early starting of weaning from four months has any effect on iron and hemoglobin levels in exclusively breastfed infants compared to no starting of weaning till the age of six months.

\section{Methods}

This cross-sectional comparative study was carried out to identify the effect of early weaning compared to no weaning on iron status among exclusively breastfed infants. The study was conducted in the outpatient department of Shahid Nazdar Bamarni primary health care center in Erbil city, Kurdistan Region, Iraq, from September 2019 to February 2020. The infants were enrolled during vaccination or well child visits of six months of age. All of them were six months old and were asymptomatic. The study sample was composed of 100 infants in two groups. Group A included 50 infants who were exclusively breastfed for four months and started weaning from four to six months with continued breastfeeding until six months (labeled as early weaning group). Group B included 50 infants who were exclusively breastfed for six months without starting weaning (labeled as no weaning group). The study inclusion criteria included all infants who were six months old, born at term, and on exclusive breastfeeding for at least four months. The study exclusion criteria were infants who were born prematurely because of deficient iron stores, ${ }^{6}$ infants who were born with small for gestational age, intrauterine growth retardation, ${ }^{14}$ and all sick infants (because serum ferritin aside from being one of the best indicators of the iron store, it is an inflammatory marker as well). ${ }^{15}$ So all children were taken when they were free of any symptoms, even simple febrile illness, to avoid the falsely elevated serum 
ferritin due to inflammatory and infectious causes. Along with the clinical evaluation, C-reactive protein (CRP) was sent for every child to interpret with and exclude the effect of any inflammation and infection. ${ }^{15-17}$ All infants after fulfilling inclusion criteria were enrolled in the study. All of them were subjected to a full history taking and thorough physical examination. A specially designed questionnaire was utilized to collect data on the infants (duration of exclusive breastfeeding, sex, mode of delivery, residency, birth weight, gestational age, singleton or twin pregnancy, current weight, and current length). All the included infants were sent for investigations, including complete blood count (CBC), serum iron, serum ferritin, and CRP Fortunately, all the iron study and hematological parameters of all participants were taken when all CRPs were negative. That is why CRP was not mentioned in the results. It was only checked as an inflammatory marker to rule out any infection in collaboration with normal white blood cells and clinical evaluation. ${ }^{15-17} \mathrm{CBC}$ was performed by mnemonic m-series. Serum ferritin and serum irons were determined by COBAS INTEGRA 400800 chemistry analyzer. All the investigations were done with the same devices using the same methods in the same laboratory throughout the duration of the study. The ethical approval of the study was granted by the research ethics committee of the
Kurdistan Board for Medical Specialties before conducting the study. Informed consent (both verbal and written) was taken from the parents of enrolled children. Statistical analysis began by entering the data on the computer using a Microsoft Excel worksheet (Excel 2017). The statistical package for the social sciences program (SPSS, version 25) was used for data analysis. The results were analyzed using frequency distribution, paired t-test, and Chi-square tests. A $P$ value of $\leq 0.05$ was considered statistically significant.

\section{Results}

More than half of all study infants were female; $58 \%$ of early-weaning and $54 \%$ of no-weaning, the vast majority $(92 \%)$ of infants in early-weaning and $(80 \%)$ of the no-weaning group were residing in urban areas. In $62 \%$ of the early-weaning and $56 \%$ of the no-weaning group, the mode of delivery was the cesarean section. There was a non-significant statistical association between study groups and the sex, residence, and mode of delivery $(P>0.05)$. There was a significant statistical association between study groups and pregnancy outcomes. All the infants of the no-weaning group and $88 \%$ of the early-weaning group were born as singleton. In contrast, $12 \%$ of early-weaning group infants were born as twin $(P=0.027)$, as shown in Table 1 .

Table 1: Basic characteristics of the study samples.

\begin{tabular}{llccc}
\hline Variable & Categories & \multicolumn{2}{c}{ Study groups } & $P$ value \\
& & Early-weaning & No-weaning \\
No. (\%) & No. (\%) & \\
\hline Sex & Male & $21(42)$ & $23(46)$ & 0.843 \\
& Female & $29(58)$ & $27(54)$ & 0.141 \\
Residence & Urban & $46(92)$ & $40(80)$ & 0.685 \\
Mode of delivery & Rural & $4(8)$ & $10(20)$ & 0.027 \\
& Cesarean section & $31(62)$ & $28(56)$ & $22(44)$ \\
Pregnancy outcome & Vaginal delivery & $19(38)$ & $50(100)$ & $0(0)$ \\
& Singleton & $44(88)$ & $50(100)$ & \\
\hline \hline
\end{tabular}


Table 2 reveals no statistically significant differences between early-weaning and no-weaning groups regarding birth weight, current length, and weight. The average birth weight of the early-weaning group was $3.13 \mathrm{~kg}$, and that of the no-weaning group was $3.27 \mathrm{~kg}$. The mean current weight of the early-weaning group was $7.18 \mathrm{~kg}$, and that of the no-weaning group was $7.34 \mathrm{~kg}$. The average length of the early-weaning group was $65.82 \mathrm{~cm}$, and that of the no-weaning group was $66.30 \mathrm{~cm}$. In all conditions, t-test was performed, and $P$ values were more than 0.05 . The findings illustrate that there was a statistically significant difference in gestational age among infants of both study groups. The infants of the no-weaning group had a higher gestational age of 39.84 weeks, while that of the early-weaning group was approximately 38 weeks $(P=0.001)$.

Table 2: Difference between "Early-weaning and No-weaning" groups in growth parameters.

\begin{tabular}{llcccc}
\hline Variables & Study-group & N & Mean & Std. Deviation & $P$ value \\
\hline Birth weight (Kg) & Early-weaning & 50 & 3.13 & 0.45 & 0.117 \\
& No-weaning & 50 & 3.27 & 0.39 & \\
Weight (Kg) & Early-weaning & 50 & 7.18 & 0.52 & 0.158 \\
& No-weaning & 50 & 7.34 & 0.58 & \\
Height (Cm) & Early-weaning & 50 & 65.82 & 1.73 & 0.279 \\
& No-weaning & 50 & 66.30 & 2.58 & \\
Gestational age (weeks) & Early-weaning & 50 & 38.08 & 0.87 & 0.001 \\
& No-weaning & 50 & 39.84 & 1.98 & \\
\hline
\end{tabular}


Table 3 demonstrates that except for white blood cells (WBC), there was a statistically significant difference in hematological parameters among participants of both study groups. The mean serum iron, ferritin, and hemoglobin were higher among early-weaning than that of no-weaning infants. The infants of the early-weaning group generally had a higher means and standard deviation of both mean corpuscular volume (MCV) and mean corpuscular hemoglobin $(\mathrm{MCH})$ in comparison to the no-weaning group. On the contrary, the RDW measure was higher for the no-weaning group (15.74\%) than the early-weaning infants (14.04\%). T-test was performed to compare the averages of the two groups, and the $P$ values were 0.001 in all conditions. However, the average platelet count was higher among no-weaning infants $(P=0.007)$.

Table 3: Difference between "Early-weaning and No-weaning" groups regarding hematological parameters.

\begin{tabular}{|c|c|c|c|c|c|}
\hline Variables & Study-group & $\mathbf{N}$ & Mean & Std. Deviation & $P$ value \\
\hline \multirow[t]{2}{*}{ Serum iron $(\mu \mathrm{g} / \mathrm{dl})$} & Early-weaning & 50 & 46.98 & 19.47 & \multirow[b]{2}{*}{0.001} \\
\hline & No-weaning & 50 & 25.66 & 9.32 & \\
\hline \multirow[t]{2}{*}{ Serum ferritin (ng/ml) } & Early-weaning & 50 & 23.94 & 25.07 & \multirow[b]{2}{*}{0.001} \\
\hline & No-weaning & 50 & 7.42 & 3.94 & \\
\hline \multirow[t]{2}{*}{ Hemoglobin (gm/dl) } & Early-weaning & 50 & 11.54 & 0.63 & \multirow[b]{2}{*}{0.001} \\
\hline & No-weaning & 50 & 10.84 & 1.15 & \\
\hline \multirow[t]{2}{*}{ MCV (fl) } & Early-weaning & 50 & 77.86 & 3.57 & \multirow[b]{2}{*}{0.001} \\
\hline & No-weaning & 50 & 71.60 & 2.85 & \\
\hline \multirow[t]{2}{*}{ MCH (pg) } & Early-weaning & 50 & 26.14 & 1.38 & \multirow[b]{2}{*}{0.001} \\
\hline & No-weaning & 50 & 25.12 & 1.13 & \\
\hline \multirow[t]{2}{*}{ RDW \% } & Early-weaning & 50 & 14.04 & 1.41 & \multirow[b]{2}{*}{0.001} \\
\hline & No-weaning & 50 & 15.74 & 1.46 & \\
\hline \multirow[t]{2}{*}{ Platelets (k/ul) } & Early-weaning & 50 & 317.60 & 38.85 & \multirow[b]{2}{*}{0.007} \\
\hline & No-weaning & 50 & 347.04 & 64.38 & \\
\hline \multirow[t]{2}{*}{ WBC (k/ul) } & Early-weaning & 50 & 8.59 & 1.51 & \multirow{2}{*}{0.240} \\
\hline & No-weaning & 50 & 8.22 & 1.60 & \\
\hline
\end{tabular}


https:/ / doi.org/ 10.15218/ zjms.2021.024

\section{Discussion}

In this study, we assessed the effects of early weaning starting from four months on iron status and hemoglobin levels in exclusively breastfed infants, as there is a controversy between the recommended duration of exclusive breastfeeding and the timing of weaning among different organizations. We found out the results of the iron study, including serum iron and serum ferritin, were significantly lower in infants who were exclusively breastfed till the age of six months (no-weaning group) compared with those who were exclusively breastfed for four months and then introduced to complementary foods from four to six months (early-weaning group). Only a few studies have investigated the effects of early weaning on iron status among exclusively breastfed infants, especially in the first six months of life. Our results turned out to agree with documented results in other studies. ${ }^{18,19}$ In our study, the mean serum ferritin was $7.42 \mathrm{ng} / \mathrm{ml}$ in the no-weaning group. In a randomized control trial study, serum ferritin was $44 \mathrm{ng} / \mathrm{ml}$ in exclusively breastfed infants at the age of six months. ${ }^{18}$ In a meta-analysis and systematic review of three randomized control trials and one observational study, the serum ferritin values were $44 \mathrm{ng} / \mathrm{ml}$ and $48.4 \mathrm{ng} / \mathrm{mlin}$ developed and developing countries, respectively, in exclusively breastfed infants at the age of six months. ${ }^{19}$ Compared to the previous studies conducted by Jonsdottir et al. ${ }^{18}$ and Qasem et al., ${ }^{19}$ serum ferritin was significantly lower in our study. The low level of serum ferritin in our study might be due to different underlining causes like deficient fetal iron stores or early umbilical cord clamping as we do not have a standard time of cord clamping and no studies have been conducted on fetal iron stores and the timing of cord clamping. In our study, serum ferritin was $23.94 \mathrm{ng} / \mathrm{ml}$ in the early-weaning group, which was higher than in the no-weaning group. In the previous two studies done by Jonsdottir et al. ${ }^{18}$ and Qasem et al., ${ }^{19}$ serum ferritin was significantly higher in the earlyweaning group with a level of $70 \mathrm{ng} / \mathrm{ml}$ in the first study, $70 \mathrm{ng} / \mathrm{ml}$ and $67.3 \mathrm{ng} / \mathrm{ml}$ in developing and developed countries, respectively. So both studies support our study regarding iron studies. According to our study, serum iron was also significantly higher in the early-weaning group compared to the no-weaning group; however, serum iron was not measured in previously mentioned studies to compare with it. The hemoglobin level in our study was $10.84 \mathrm{gm} / \mathrm{dl}$ in the no-weaning group and $11.54 \mathrm{gm} / \mathrm{dl}$ in the early-weaning group. Jonsdottir et al. concluded that there was no significant difference in hemoglobin levels between early-weaning and no-weaning groups. ${ }^{18}$ Qasem et al. demonstrated no significant difference in hemoglobin level between two study groups in developed countries, but the hemoglobin level was significantly higher with a value of $10.9 \mathrm{gm} / \mathrm{dl}$ in the early-weaning group and $10.4 \mathrm{gm} / \mathrm{dl}$ in the no-weaning group in developing countries. ${ }^{19}$ In our study, the red blood cell indices, including $\mathrm{MCV}$ and $\mathrm{MCH}$, were $71.60 \mathrm{fl}$ and $25.12 \mathrm{pg}$, respectively, in the no-weaning group, which were significantly lower compared to $77.86 \mathrm{fl}$ and $26.14 \mathrm{pg}$ in the early-weaning group, respectively. In contrast, the red cell distribution width (RDW) was higher in the no-weaning group $(15.74 \%)$ compared to the early-weaning group (14.04\%), which all supported the diagnosis of iron deficiency anemia in the no-weaning group. Jonsdotir et al. no significant difference between the two study groups (early-weaning and no-weaning group) regarding MCV and RDW. ${ }^{18}$ Qasem et al. did not mention these parameters between the two study groups in their study. ${ }^{19}$ Such findings of the iron study and hematological parameters were in concordance with the results of Qasem et al. and Jonsdotir et al., but its deficient in serial follow up of the iron and hemoglobin of these infants. Few other studies supported our findings as well. A study 
carried out by Krishnaswamy et al. concluded that the prevalence of iron deficiency was gradually increasing from three months toward four and five months with $5.4 \%, 21.4 \%$, and $36.4 \%$ and iron deficiency anemia in these age groups were $4.6 \%, 16.7 \%$, and $11.4 \%$, respectively. ${ }^{20}$ Another study showed that the prevalence of iron deficiency and iron deficiency anemia were $3.7 \%$ and $2.7 \%$, respectively, in infants aged 4 to 6 months and predominantly breastfed; hence it advised the complementary foods to be started after four months. ${ }^{21}$ Few recent studies by Raj et al. ${ }^{22}$ and Eneroth et al., ${ }^{13}$ contradict these findings as they concluded that exclusive breastfeeding for six months did not associate with iron deficiency since their iron stores were normal at six months of age. Regarding the limitations of the study, our study was a small population based study that included only 100 infants with no previous hematological parameters. Ideally, it was better to measure their hematological parameters at age of four months when both groups were exclusively breastfed. Also, the groups were not matched, and the study might be prone to biases of the effects of any associated factors like sex, mode of delivery, birth weight, and gestational age. Further studies are required with a large sample size and matched associated factors to know the exact effect of early weaning on iron status among exclusively breastfed infants.

\section{Conclusion}

In the present study, we concluded that exclusive breastfeeding for six months without weaning is associated with both iron deficiency and iron deficiency anemia at six months of age. Consequently, early weaning had a great role in the prevention of both iron deficiency and iron deficiency anemia in exclusively breastfed infants.

\section{Competing interests}

The authors declare no competing interests.

\section{References}

1. Lessen R, Kavanagh K. Position of the academy of nutrition and dietetics: promoting and supporting breastfeeding. J Acad Nutr Diet 2015; 115(3):444-9.

2. Haimi M, Lerner A. Nutritional deficiencies in the pediatric age group in a multicultural developed country, Israel. World J Clin Cases 2014; 2(5):120-5.

3. Camaschella C. Iron-deficiency anemia. N Engl J Med 2015; 372(19):1832-43.

4. Berglund S, Domellöf M. Meeting iron needs for infants and children. Curr Opin Clin Nutr Metab Care 2014; 17(3):267-72.

5. Qasem WA, Friel JK. An overview of iron in term breast-fed infants. Clin Med Insights Pediatr 2015; 9:79-84.

6. Baker RD, Greer FR. Committee on Nutrition American Academy of Pediatrics. Diagnosis and prevention of iron deficiency and iron-deficiency anemia in infants and young children (0-3 years of age). Pediatrics 2010; 126(5):1040-50.

7. Hewa FZ, Zangana KO. Influence of iron deficiency anemia on growth: A cross-sectional study. Medical Journal of Babylon 2019; 16(01):335.

8. Ziegler EE, Nelson SE, Jeter JM. Iron stores of breastfed infants during the first year of life. Nutrients 2014; 6(5):2023-34.

9. Finkelstein JL, O'Brien KO, Abrams SA, Zavaleta $\mathrm{N}$. Infant iron status affects iron absorption in Peruvian breastfed infants at 2 and 5 months of age. Am J Clin Nutr 2013; 98(6):1475-84.

10. Burke RM, Rebolledo PA, Aceituno AM, Revollo $R$, IñiguezV, Klein $M$, et al. Effect of infant feeding practices on iron status in a cohort study of Bolivian infants. BMC Pediatr 2018; 18(1):107.

11. Wang $F$, Liu H, Wan Y, Li J, Chen Y, Zheng J, et al. Prolonged Exclusive Breastfeeding Duration Is Positively Associated with Risk of Anemia in Infants Aged 12 Months. J Nutr 2016; 146(9):1707-13.

12. Ziegler EE, Nelson SE, Jeter JM. Iron supplementation of breastfed infants. Nutr Rev 2011; 69(s1):S71-7.

13. Eneroth $H$, El Arifeen S, Persson LA, Kabir L, Lonnerdal B, Hossain MB, et al. Duration of exclusive breast-feeding and infant iron and zinc status in rural Bangladesh. J Nutr 2009; 139(8):1562-7.

14. Kim HA, Park SH, Lee EJ. Iron status in small for gestational age and appropriate for gestational age infants at birth. Korean J Pediatr 2019; 62(3):102-7.

15. Thurnham DI, McCabe LD, Haldar S, Wieringa FT, Northrop-Clewes CA, McCabe GP. Adjusting plasma ferritin concentrations to remove the effects of subclinical inflammation in the assessment of iron deficiency: a metaanalysis. Am J Clin Nutr 2010; 92(3):546-55. 
16. Namaste SM, Rohner F, Huang J, Bhushan NL, Flores-Ayala R, Kupka R, et al. Adjusting ferritin concentrations for inflammation: Biomarkers Reflecting Inflammation and Nutritional Determinants of Anemia (BRINDA) project. Am J Clin Nutr 2017; 106(Suppl 1):359S-71.

17. Sproston NR, Ashworth JJ. Role of C-reactive protein at sites of inflammation and infection. Front Immunol 2018; 9:754.

18. Jonsdottir $\mathrm{OH}$, Thorsdottir I, Hibberd PL, Fewtrell MS, Wells JC, Palsson GI, et al. Timing of the introduction of complementary foods in infancy: A randomized controlled trial. Pediatrics 2012; 130(6):1038-45.

19. Qasem W, Fenton T, Friel J. Age of introduction of first complementary feeding for infants: a systematic review. BMC Pediatr 2015; 15:107.

20. Krishnaswamy S, Bhattarai D, Bharti B, Bhatia P, Das $R$, Bansal $D$. Iron deficiency and iron deficiency anemia in 3-5 months-old, breastfed healthy infants. Indian J Pediatr 2017; 84(7):5058.

21. Chen CM, Mu SC, Shih CK, Chen YL, Tsai LY, Kuo YT, et al. Iron status of infants in the first year of life in Northern Taiwan. Nutrients 2020; 12(1):139.

22. Raj S, Faridi M, Rusia U, Singh O. A prospective study of iron status in exclusively breastfed term infants up to 6 months of age. Int Breastfeed $J$ 2008; 3:3. 